\title{
TWO VISUAL PIGMENTS IN A SINGLE PHOTORECEPTOR CELL: IDENTIFICATION AND HISTOLOGICAL LOCALIZATION OF THREE mRNAS ENCODING VISUAL PIGMENT OPSINS IN THE RETINA OF THE BUTTERFLY PAPILIO XUTHUS
}

\author{
JUNKO KITAMOTO ${ }^{1}$, KATSUHIKO SAKAMOTO ${ }^{1}$, KOICHI OZAKI ${ }^{2}$, YUJI MISHINA ${ }^{3, *}$ \\ AND KENTARO ARIKAWA ${ }^{1,4, \dagger}$ \\ ${ }^{1}$ Graduate School of Integrated Science, Yokohama City University, 22-2 Seto, Kanazawa-ku, Yokohama 236-0027, \\ Japan, ${ }^{2}$ Graduate School of Science, Osaka University, 1-1 Machikaneyama-cho, Toyonaka 560-0043, Japan, \\ ${ }^{3}$ University of Texas, MD Anderson Cancer Center, 1515 Holcombe Blvd, Houston, TX77030, USA and ${ }^{4}$ PRESTO, \\ Japan Science and Technology Corporation, Japan \\ *Present address: Laboratory of Reproductive and Developmental Toxicology, National Institute of Environmental Health Sciences, PO Box \\ 12233, Research Triangle Park, NC 27709, USA \\ $\dagger$ Author for correspondence (e-mail: arikawa@yokohama-cu.ac.jp).
}

Accepted 27 January; published on WWW 20 April 1998

This paper describes the localization of newly identified visual pigment opsins in the tiered retina of the Japanese yellow swallowtail Papilio xuthus. We first cloned three cDNAs encoding visual pigment opsins, PxRh1, PxRh2 and PxRh3, and then carried out histological in situ hybridization to localize their mRNAs in the retina. By combining the present data with our previous electrophysiological results, we concluded that both PxRh1 and PxRh2 correspond to visual pigments expressed in photoreceptor cells sensitive in the green wavelength region (green receptors), whereas PxRh3 corresponds to a pigment in red receptors. The in situ hybridization studies showed that some photoreceptor cells express two opsin mRNAs. In the ventral half of the eye, all green receptors in the distal tier were labelled by both PxRh1 and PxRh2 probes. The labelling by the PxRh2 and PxRh3 probes was detected throughout the eye in the proximal tier; in $18 \%$ of ommatidia, the probes labelled the same photoreceptor cell. These results suggest that the possible co-localization of two different visual pigments will broaden the sensitivity spectrum of the photoreceptor cells.

Key words: visual pigment, mRNA, opsin, butterfly, vision, Papilio xuthus, compound eye, color vision, retina insect, ommatidium.

\section{Introduction}

The Japanese yellow swallowtail Papilio xuthus has a complex retina with at least five types of photoreceptor cell, each of which possesses a different spectral sensitivity. The sensitivities peak in the ultraviolet, violet, blue, green and red wavelength regions (Arikawa et al. 1987). We have demonstrated electrophysiologically that the anatomically unique set of nine photoreceptor cells in an ommatidium, R1-R9 (Fig. 1), are spectrally heterogeneous. R1 and R2 are ultraviolet, violet or blue receptors, R3 and R4 are green receptors (Bandai et al. 1992) and R5-R9 are either green or red receptors (Arikawa and Uchiyama, 1996). These results indicate that the ommatidia are not identical to each other in terms of their spectral receptor composition. A single ommatidium can contain a minimum of two (e.g. R1,2, ultraviolet; R3-R9, green) and a maximum of four (e.g. R1, ultraviolet; R2, blue; R3,4, green; R5-R9, red) types of spectral receptor.

There are a number of questions that are hard to answer electrophysiologically, however. For instance, do all R5-R9 cells in a single ommatidium have the same sensitivity spectrum, and how are the different types of ommatidium arranged in the retina? As a more direct approach to these problems, we conducted a molecular biological analysis to characterize the visual pigment opsins of $P$. xuthus.

The spectral sensitivity of a photoreceptor cell is primarily determined by the absorption spectrum of the visual pigment in the cell. The visual pigment is a membrane-bound protein, opsin, with a chromophore attached. In P. xuthus, the chromophore is 11-cis 3-hydroxyretinal: no other chromophores have been identified (Seki et al. 1987). This indicates that the difference in absorption spectrum of visual pigments should be attributed to the structure of opsin. We first cloned cDNAs encoding visual pigment opsins from a cDNA library of the retina of $P$. xuthus. We then determined the primary structure of three visual pigment opsins and we carried out histological in situ hybridization to localize the opsin mRNAs in the retina. The in situ hybridization clearly indicated that some photoreceptor cells contain two different opsin mRNAs and that there are at least three types of ommatidium randomly distributed in the eye. 


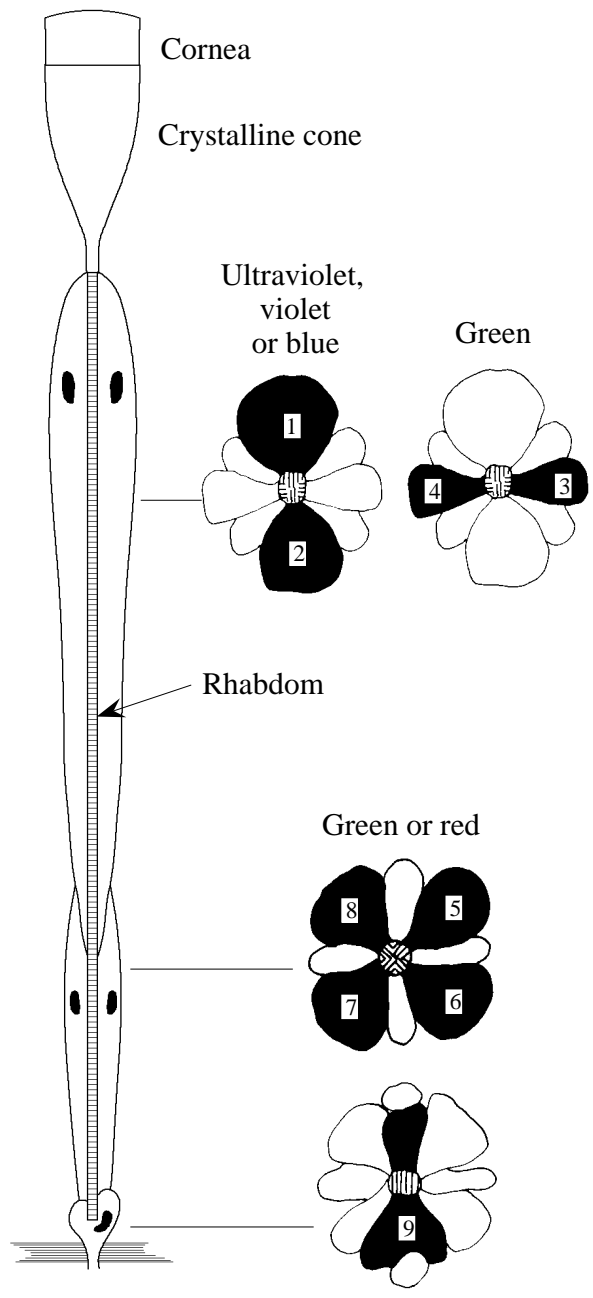

Fig. 1. Schematic representation of a Papilio xuthus ommatidium. (Left) Longitudinal view. The rhabdom is of the fused and tiered type. The distal tier is made up of the microvilli of four distal cells (R1-R4). The other cells (R5-R9) form the proximal tier; the bilobed R9 cell contributes only at the base of the rhabdom. (Right) Transverse sectional view. The spectral type of each photoreceptor cell is indicated, based on our previous studies (Arikawa and Uchiyama, 1996; Bandai et al. 1992).

\section{Materials and methods} Animals

The Japanese yellow swallowtail Papilio xuthus Linnaeus was held as a laboratory stock culture. We used individuals of both sexes emerged from non-diapausing pupae.

\section{Molecular cloning}

Retinal mRNA was extracted from frozen compound eyes by using the QuickPrep mRNA purification kit (Pharmacia). Partial sequences of $P$. xuthus opsin mRNAs were amplified by the reverse transcriptive polymerase chain reaction (RTPCR) with two oligonucleotide primers, F5 and R8, originally designed on the basis of the Drosophila and octopus opsins to amplify invertebrate opsin cDNAs (Hariyama et al. 1993). The RT-PCR-amplified fragments were subcloned into pBluescriptII KS(-) plasmid vector and sequenced. We thus identified fragments of three opsin-like sequences.

We constructed a cDNA library of the P. xuthus retina by using the $\lambda$ ZAPII cDNA synthesis kit (Stratagene). We screened the library with the RT-PCR-amplified fragments. Phage plaques were transferred to nylon membranes (Hybond $\mathrm{N}$, Amersham), which were pre-incubated in a hybridization solution (50\% formamide) at $53^{\circ} \mathrm{C}$ for $1 \mathrm{~h} .{ }^{32} \mathrm{P}$-labelled DNA probes were then added, and the membranes were incubated overnight at $53^{\circ} \mathrm{C}$. After washing the membranes with $0.2 \times \mathrm{SSC}, 0.1 \% \mathrm{SDS}$ at $60^{\circ} \mathrm{C}$, positive clones were identified using BAS 2000 (Fuji Film). The cDNA inserts excised from the clones were reacted for sequencing using the sequiTherm cycle sequencing kit (Epicentre technologies) and sequenced with LIC-4000 (LI-COR).

\section{Comparison of amino acid sequences}

To compare the sequences of the cloned cDNAs of $P$. xuthus with other insect opsins, deduced amino acid sequences were aligned using an alignment program (CLUSTAL W 1.6), and then a phylogenetic analysis was performed using the neighbour-joining method (PHYLIP 3.572) with bovine opsin as an outgroup.

\section{In situ hybridization}

Isolated eyes were fixed in $4 \%$ paraformaldehyde in $0.1 \mathrm{moll}^{-1}$ sodium phosphate buffer $(\mathrm{pH} 7.2, \mathrm{~PB})$ for $0.5-2 \mathrm{~h}$ at $25^{\circ} \mathrm{C}$ and preserved in $30 \%$ sucrose in PB at $4{ }^{\circ} \mathrm{C}$ overnight. Eyes were then embedded and frozen in Tissue-Tec OCT compound (Miles) and sectioned at $8 \mu \mathrm{m}$ thickness with a cryostat. Sections were mounted on poly-L-lysine-coated slides.

Probes for in situ hybridization were designed to hybridize to approximately 200 bases of the mRNAs at the non-coding region downstream of the $\mathrm{C}$ terminus. Corresponding cDNA regions were first subcloned into pGEM-3zf(+) vector, and digoxigenin (DIG)-labelled cRNAs were then generated using a DIG-RNA labelling kit (Boehringer, Mannheim). The absence of cross hybridization was confirmed by dot blot analysis.

Sections were dehydrated in a graded ethanol series and dried at $65^{\circ} \mathrm{C}$ for $1 \mathrm{~h}$. The sections were treated with hybridization solution containing $0.5 \mu \mathrm{g} \mathrm{ml}^{-1}$ cRNA probe at $65{ }^{\circ} \mathrm{C}$ for $16 \mathrm{~h}$ (for details, see Sakamoto et al. 1996). The probes were further visualized by anti-DIG immunocytochemistry (DIG nucleic acid detection kit, Boehringer Mannheim).

\section{Results}

Primary structure of three opsins in the Papilio xuthus retina

We identified three opsin-like sequences. All were approximately $1.6 \mathrm{~kb}$ in length, containing an open reading frame of $1137 \mathrm{bp}$, approximately $73 \%$ of which were identical. 


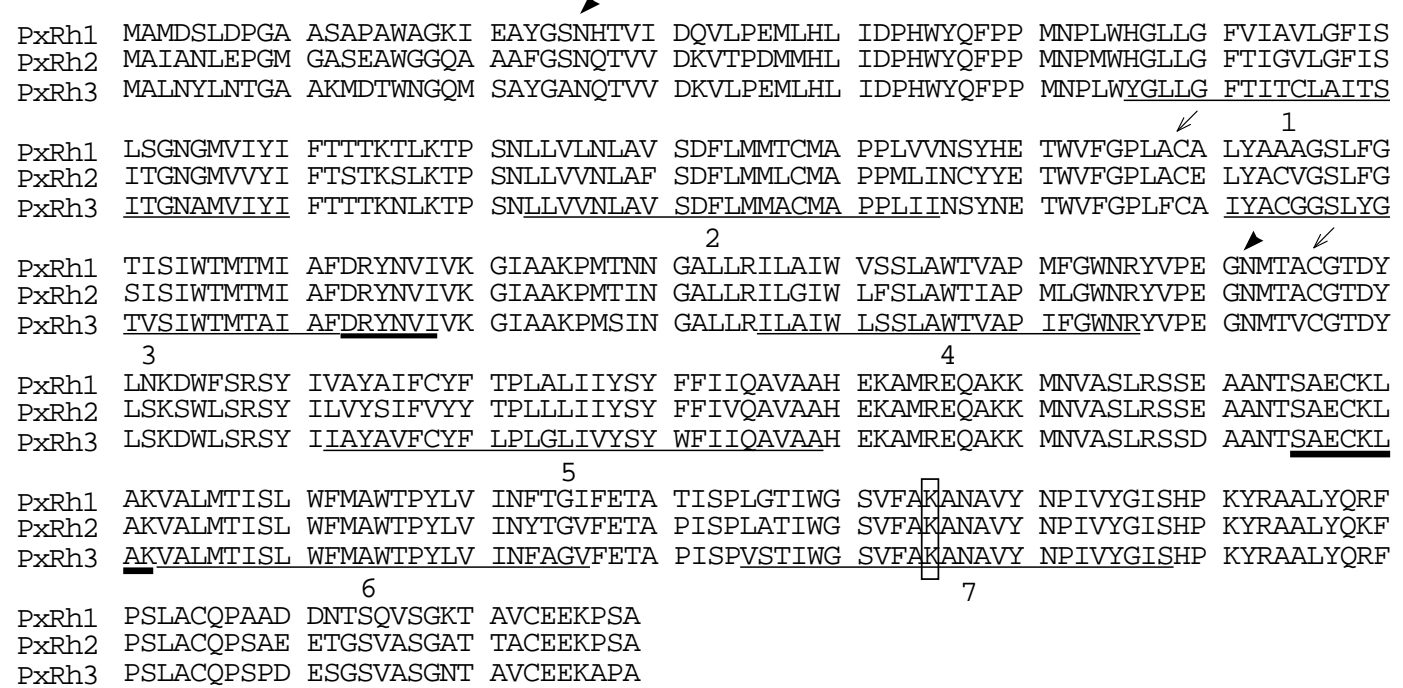

Fig. 2. Amino acid sequences of Papilio xuthus opsins PxRh1, PxRh2 and PxRh3. Underlining indicates transmembrane helices (1-7). Bold underlining indicates sites for binding of G-proteins. Arrowheads show possible glycosylation sites. Arrows show sites for disulphide bonding. The framed area is the site for chromophore attachment.

Deduced amino acid sequences indicate that all three sequences consist of 379 amino acid residues (Fig. 2). We hereafter refer to these peptides as PxRh1 (Papilio xuthus rhodopsin 1), PxRh2 and PxRh3, respectively.

We confirmed their identity as opsins by comparing their amino acid sequences with those of other opsins. It appears that they share a distinct homology. Hydropathy analysis revealed seven transmembrane helices. Conserved amino acids are Lys-325 for chromophore attachment, Cys-129 and Cys206 for disulphide bonding and Asn-26 and Asn-202 for glycosylation (Fig. 2). The cytoplasmic loops contain sequences that presumably interact with G-proteins.

Fig. 3 represents the molecular phylogenetic relationship between $P$. xuthus opsins and other insect opsins. The insect opsins are divided into two groups: one that includes the Drosophila $\mathrm{Rh} 1$ and $\mathrm{Rh} 2$ types and which is tuned to longwavelength light, and a second containing Drosophila $\mathrm{Rh} 3$ and $\mathrm{Rh} 4$, which is tuned to short-wavelength light. All of the three $P$. xuthus opsins form a single cluster within the first group. Other opsins tuned to short-wavelength light should be present, but these were not detected in the present study. Other opsins tuned to short-wavelength light should be present. In fact, we have already determined partial sequences of two opsins clustered in the second group (J. Kitamoto, unpublished observation).

\section{Opsin distribution in the retina}

To reveal the distribution of these opsin mRNAs in the retina, we performed histological in situ hybridizations (Figs 4-6). The eyes were cut parallel to the longitudinal axis of the ommatidia, showing an array of ommatidia from the dorsal to the ventral edge ( $\mathrm{A}$ and $\mathrm{D}$ in each figure), or were cut transversely, at different depths (B, C and $\mathrm{E}$ in each figure).

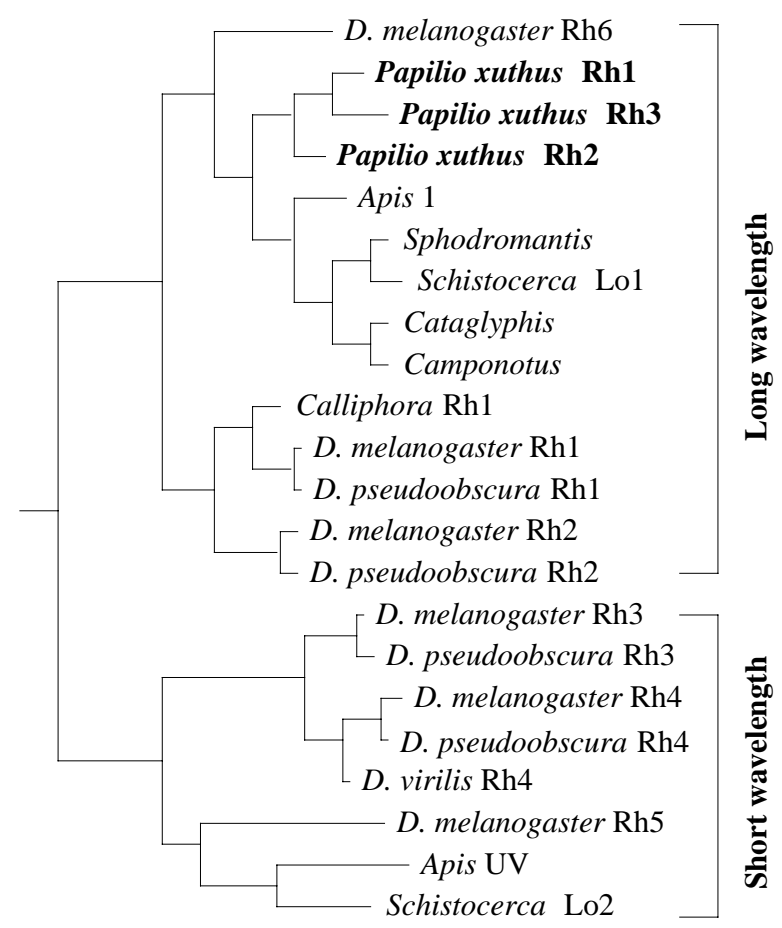

Fig. 3. Phylogenetic tree of insect opsins. Papilio xuthus opsins cluster in the group of opsins tuned to long-wavelength light. Data accession numbers: Apis 1, U26026; Apis ultraviolet, U70841; Calliphora Rh1, M58334; Camponotus, U32502; Cataglyphis, U32501; D. melanogaster Rh1, A90864; D. melanogaster Rh2, A24058; D. melanogaster Rh3, Y00043; D. melanogaster Rh4, M17730; D. melanogaster Rh5, U80667; D. melanogaster Rh6, Z86118; D. pseudoobscura Rh1, X65877; D. pseudoobscura Rh2, X65878; D. pseudoobscura Rh3, X65879; D. pseudoobscura Rh4, $\mathrm{X} 65880 ;$ D. virilis Rh4, M77281; Schistocerca Lo1, X80071; Schistocerca Lo2, X80072; Sphodromantis, X71665. 

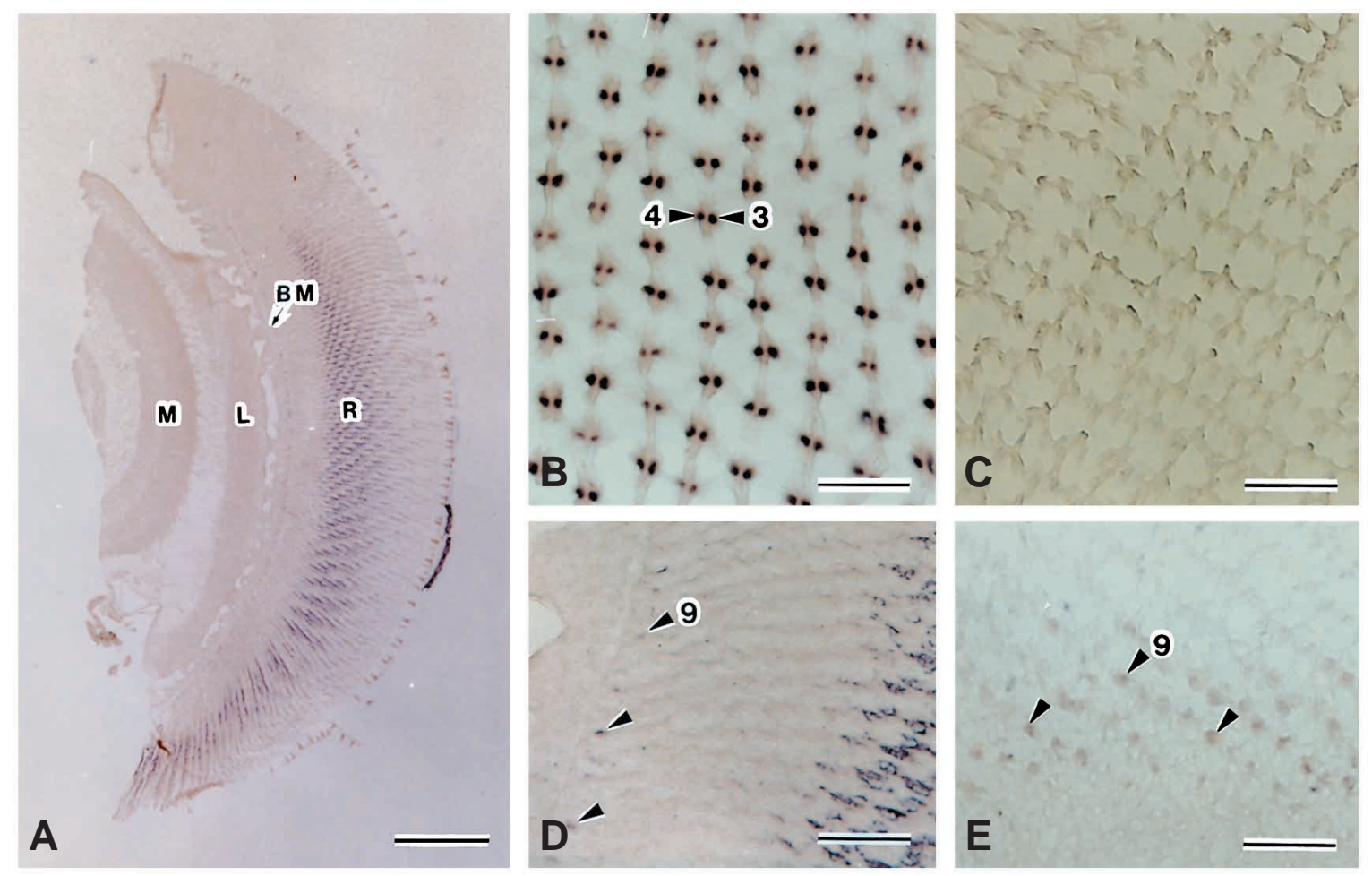

Fig. 4. PxRh1 in situ hybridization. (A) Longitudinal view of ommatidia from the dorsal (up) to the ventral (bottom) edge. Labelling is evident in the ventral half of the retina. (B) Transverse section through the distal tier in the ventral region of the eye. R3 and R4 are labelled (arrowheads). (C) Transverse section through the proximal tier. (D) Enlargement of A, showing R9 photoreceptors labelled (arrowheads). (E) Transverse section close to the basement membrane, showing R9 cells labelled (arrowheads). BM, basement membrane; L, lamina; M, medulla; R, retina. Scale bars, $250 \mu \mathrm{m}(\mathrm{A}), 25 \mu \mathrm{m}(\mathrm{B}-\mathrm{E})$.

The localization of corresponding mRNA was visualized as a dark deposit.

The PxRh1 probe hybridized to all ommatidia except for the dorsal region of the eye (Fig. 4A). The border between the unlabelled and labelled regions corresponds to the part of the eye viewing slightly above the horizon, and these two regions are hereafter referred to as the dorsal and the ventral halves, although the ventral half contains more ommatidia. In the ventral half, the PxRh1 probe clearly labelled R3 and R4, two of the four distal photoreceptor cells (Fig. 4B). Weak signals were observed for R9 cells (Fig. 4D,E). R9 cells are very small, and we have therefore been unable to determine unequivocally whether all R9 cells in the ventral half were labelled. R9 cells in the dorsal half were not labelled.

The PxRh2 probe gave strong labelling throughout the retina from the dorsal to the ventral edge (Fig. 5A). In the distal tier, the probe labelled R3 and R4 photoreceptors in all ommatidia (Fig. 5B). In the proximal tier, R5-R8 cells were labelled in $52 \%$ of ommatidia; R5-R8 cells were not labelled in the remaining ommatidia (Fig. 5C). Some R9 cells were clearly labelled (Fig. 5D,E) but, again, it was not possible to establish whether all R9 cells were labelled.

PxRh3 labelling was found only in the proximal tier (Fig. 6A), where it labelled R5-R8 cells in $66 \%$ of ommatidia (Fig. 6C). R9 cells were not labelled (Fig. 6D,E).
PxRh2 and PxRh3 labelling overlapped in approximately $18 \%$ of ommatidia. Fig. 7 shows two adjacent sections each labelled with either the PxRh2 (Fig. 7A) or the PxRh3 (Fig. 7B) probe. Although these two probes labelled different populations of ommatidia, in this particular set of pictures the R5-R8 cells of 21 of the 97 ommatidia (21.6\%) were doublelabelled (orange-marked). Table 1 summarizes the labelling results in R5-R8 cells. A $\chi^{2}$-test showed that the arrangement of three types of ommatidia was random (Arikawa and Stavenga, 1997; Mollon and Bowmaker, 1992).

\section{Discussion}

Spectral properties of cloned opsins

By combining the present results with our previous

Table 1. Labelling results in the proximal $R 5-R 8$ photoreceptors

\begin{tabular}{lcc}
\hline & $\begin{array}{c}\text { Number of } \\
\text { ommatidia }\end{array}$ & $\begin{array}{c}\text { Percentage of } \\
\text { ommatidia }\end{array}$ \\
\hline PxRh2 only & 421 & 34.4 \\
PxRh2 + PxRh3 & 217 & 17.7 \\
PxRh3 only & 587 & 47.9 \\
Total & 1225 & 100 \\
\hline
\end{tabular}



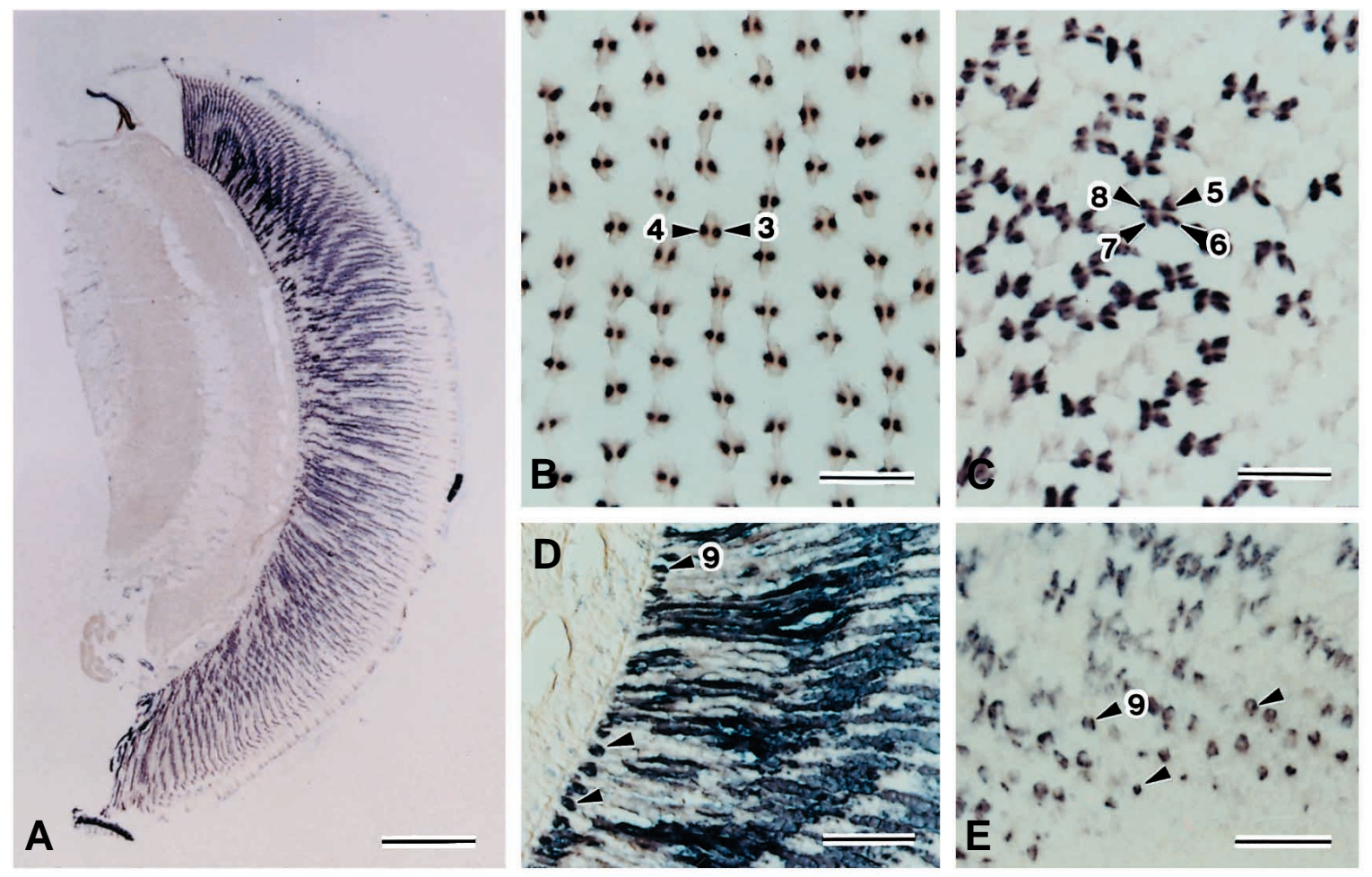

Fig. 5. PxRh2 in situ hybridization. (A) Labelling is evident throughout the retina, in both the distal and proximal tiers. (B) Transverse section through the distal tier. R3 and R4 photoreceptors are labelled (arrowheads). (C) Transverse section through the proximal tier. R5-R8 cells in some ommatidia are labelled (arrowheads). (D) Enlargement of A, showing R9 cells labelled (arrowheads). (E) Transverse section through R9 cells (arrowheads). Scale bars, $250 \mu \mathrm{m}$ (A), $25 \mu \mathrm{m}$ (B-E).
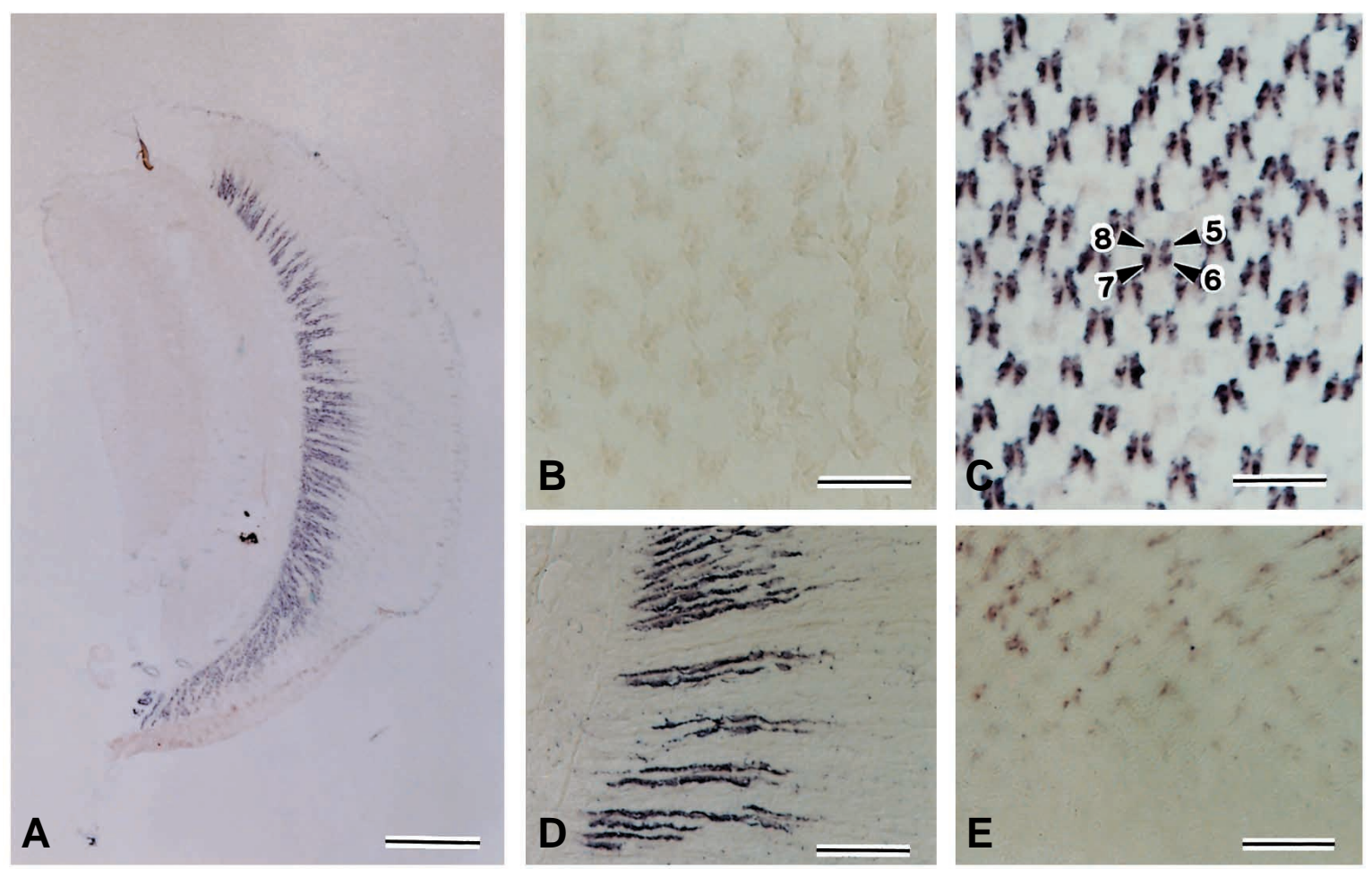

Fig. 6. PxRh3 in situ hybridization. (A) Longitudinal section. Labelling is evident throughout the retina, but only in the proximal tier. (B) Transverse section through the distal tier. (C) Transverse section through the proximal tier. R5-R8 photoreceptors in some ommatidia are labelled (arrowheads). (D) Enlargement of A, showing that R9 cells are not labelled. (E) Transverse section through R9 cells. Scale bars, $250 \mu \mathrm{m}(\mathrm{A}), 25 \mu \mathrm{m}(\mathrm{B}-\mathrm{E})$. 


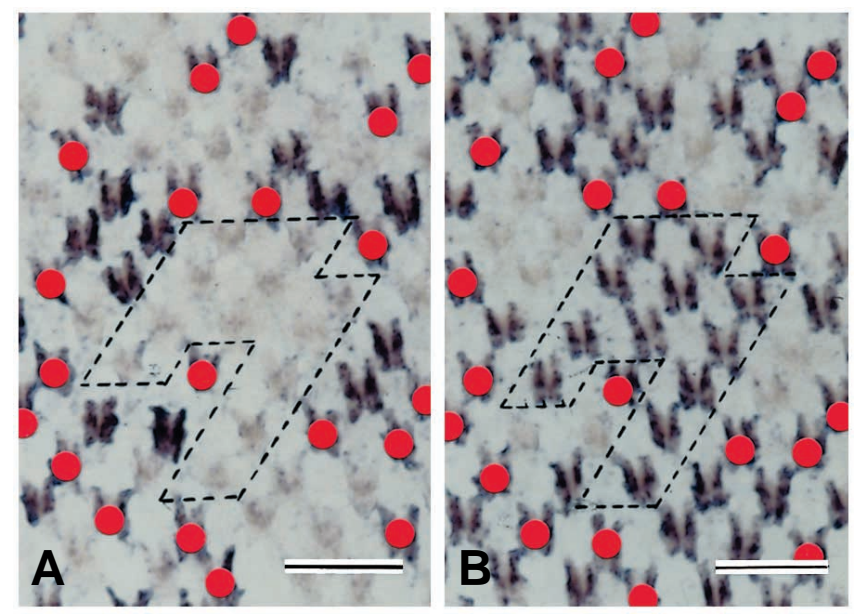

Fig. 7. Labelling with the PxRh2 probe (A) and the PxRh3 probe (B) in adjacent sections. Framed areas indicate the same set of ommatidia. The labelling patterns obtained using these probes overlap in some ommatidia (orange-marked). Scale bars, $25 \mu \mathrm{m}$.

electrophysiological results (Arikawa and Uchiyama, 1996; Bandai et al. 1992), we predicted the absorption spectra of the visual pigments as follows (Table 2). Of the four distal cells, R3 and R4 are green receptors (Fig. 1) (Bandai et al. 1992). These green receptors express PxRh1 (Fig. 4B) and PxRh2 (Fig. 5B), at least in the ventral half of the eye, indicating that both PxRh1 and PxRh2 correspond to green-sensitive visual pigments. PxRh3 labelling was found only in the proximal tier, where R5-R8 cells in $66 \%$ of ommatidia appeared to express PxRh3. The PxRh3-labelled R5-R8 cells are probably red receptors, because (1) red receptors are found exclusively in the proximal tier and (2) approximately $75 \%$ of R5-R8 cells are red-sensitive (Arikawa and Stavenga, 1997; Arikawa and Uchiyama, 1996). Although these figures are not a perfect match, the percentage of PxRh3-bearing ommatidia (66\%) is larger, i.e. closer to $75 \%$, than that of the PxRh2-bearing ommatidia (53\%).

It would be hard to show using electrophysiology alone whether the R5-R8 cells in a single ommatidium are always

Table 2. Correlation of in situ hybridization with electrophysiological data

\begin{tabular}{llccc}
\hline \multirow{2}{*}{$\begin{array}{l}\text { Cell } \\
\text { number }\end{array}$} & $\begin{array}{c}\text { Electrophysiological } \\
\text { spectral type* }\end{array}$ & \multicolumn{3}{c}{ In situ hybridization } \\
\cline { 3 - 5 } & \multicolumn{2}{c}{ PxRh1† } & PxRh2 & PxRh3 \\
\hline R1, R2 & Ultraviolet, violet or blue & - & - & - \\
R3, R4 & Green & + & + & - \\
R5-R8 & Green or red & - & $+\neq$ & $+\$$ \\
R9§ & Green or red & + & + & -
\end{tabular}

*Bandai et al. (1992); Arikawa and Uchiyama (1996).

$\dagger$ Labelling only in the ventral half of the retina.

$\ddagger$ Labelling of PxRh2 and PxRh3 probes overlaps in $18 \%$ of ommatidia.

§Some R9 cells are double-labelled. identical. In situ hybridization answers this question clearly. R5-R8 cells in one ommatidium were always labelled simultaneously, either by both the PxRh2 and PxRh3 probes or by one of them. This means that the sensitivity spectra of the R5-R8 cells of an ommatidium are identical.

The situation in the basal R9 photoreceptor cells is still unresolved. Our electrophysiological results indicated that R9 cells are either green- or red-sensitive (Arikawa and Uchiyama, 1996). However, R9 cells were labelled with the PxRh1 and/or $\mathrm{PxRh} 2$ probes, but not with the PxRh3 probe, and some R9 cells appeared to be double-labelled with both PxRh1 and PxRh2. In any case, the R9 cells containing PxRh1 and/or $\mathrm{PxRh} 2$ are probably green-sensitive. There should be another population of red-sensitive R9 cells, although the PxRh3 probe did not appear to label any R9 cells. The simplest explanation for this observation is that the red-sensitive R9 cells contain another uncloned visual pigment. In fact, Briscoe (1998) recently reported partial amino acid sequences of six opsins from Papilio glaucus (PglRh1-PglRh6), four of which (PglRh1-PglRh4) appeared to be long wavelength opsins. PglRh1-PglRh3 are almost identical to PxRh1-PxRh3, at least in the limited regions reported (approximately 80 amino acids). PglRh4 may be the counterpart of the uncloned opsin expressed in the red-sensitive R9 of $P$. xuthus.

\section{Double expression of opsins in single photoreceptor cells}

A conspicuous feature of the $P$. xuthus retina revealed here is the existence of photoreceptor cells that were doublelabelled by two different probes. The R3 and R4 photoreceptor cells in the ventral half of the retina (Figs 4B, 5B) were labelled with both the PxRh1 and PxRh2 probes. The PxRh2 and PxRh3 probes simultaneously labelled the R5-R8 photoreceptor cells in approximately $18 \%$ of ommatidia (Fig. 7; Table 1). Such double labelling strongly implies that these photoreceptor cells bear two different visual pigments.

The functional significance of the co-localization of two opsins in single photoreceptor cells has yet to be established. A possible function of having two visual pigments, especially with different spectral absorbances, is to broaden the sensitivity spectrum. In fact, we recently identified a population of R5-R8 photoreceptor cells with a very broad sensitivity spectrum peaking at approximately $500 \mathrm{~nm}$ with a half-bandwidth of approximately $170 \mathrm{~nm}$ (K. Arikawa, unpublished observations). The broad sensitivity spectrum may be attributable to co-localization of two visual pigments encoded by PxRh2 and PxRh3 mRNAs. It has been shown in Drosophila melanogaster that photoreceptor cells in which two different visual pigments are artificially co-expressed display the summed sensitivity spectrum of the individual pigments (Feiler et al. 1992).

According to the standard concept in visual physiology, a single photoreceptor cell bears only a single type of visual pigment. Clearly, however, this concept needs adjustment. Co-localization of two opsins in a single photoreceptor cell has been reported in fish and in some rodents. In fish, developing photoreceptor cells in the retinal ridge express 
multiple opsin mRNAs, but only one of them will become the mRNA present when the cells mature (Satoh et al. 1995). This has been explained as a phenomenon that occurs during development. In the retina of adult rodents, some photoreceptor cells around the border of two retinal fields each dominated by cones contain different visual pigments (Röhlich et al. 1994). This co-expression 'may be due to the overlapping of regulatory factors determining' (Röhlich et al. 1994) the two retinal fields. However, such an explanation is not applicable to the $P$. xuthus retina, because the doublelabelled and single-labelled ommatidia are randomly distributed. Another example of double expression of visual pigment is seen in the crab Hemigrapsus sanguineus, where seven of the eight photoreceptor cells in all ommatidia coexpress two opsin mRNAs (Sakamoto et al. 1996). The present case of $P$. xuthus is the clearest example of multiple expression of opsin mRNAs in single photoreceptor cells. Although the pattern of the double labelling is very clear, there is still a possibility that only one of the two mRNAs is translated into visual pigment. Immunocytochemical studies using specific antibodies, which are now in progress, will clarify this point.

\section{Both regularity and randomness occur in the retina}

The labelling pattern in the R5-R8 proximal photoreceptor cells clearly indicates that there are at least three types of ommatidia (Table 1). We hypothesize that they correspond to the three optically identifiable types of ommatidium, i.e. the red-pigmented, red-pigmented plus fluorescence, and yellowpigmented ommatidia. The overall organization of the $P$. xuthus retina is a random mesh of these types (Arikawa and Stavenga, 1997).

The labelling pattern with the PxRh2 probe revealed another conspicuous feature (Fig. 5). In the distal tier, the probe labelled R3 and R4 cells in all ommatidia and demonstrates beautifully the hexagonal arrangement of the ommatidia (Fig. 5B). This is in sharp contrast to the random organization in the proximal tier (Fig. 5C). The regular, hexagonal array of the R3-R4 cell system is, of course, ideal for spatial resolution, while the random array in the proximal tier may be related to colour vision, perhaps being required to avoid chromatic aliasing and/or to improve contrast vision (Mollon and Bowmaker, 1992). The following hypothesis can thus be proposed: the $P$. xuthus retina is functionally duplex, with the regularly arranged system for spatial resolution and the randomly arranged system for colour vision. In any case, the
$P$. xuthus retina will be a good model for studying the functional significance of the receptor mosaic in general.

We thank D. G. Stavenga for critical reading of the manuscript. Mariko Hirokawa provided technical assistance in the initial stages of the work. This work was supported by grants from the Japan Science Society and the Circle for the Promotion of Science and Engineering to J.K. and from the Ministry of Education, Science and Culture of Japan to K.A. DDBJ/EMBL/GenBank accession numbers: AB007423 (PxRh1), AB007424 (PxRh2), AB007425 (PxRh3).

\section{References}

Arikawa, K., Inokuma, K. AND Eguchi, E. (1987). Pentachromatic visual system in a butterfly. Naturwissenschaften 74, 297-298

ARIKAWA, K. AND Stavenga, D. G. (1997). Random array of colour filters in the eyes of butterflies. J. exp. Biol. 200, 2501-2506.

Arikawa, K. And Uchiyama, H. (1996). Red receptors dominate the proximal tier of the retina in the butterfly Papilio xuthus. J. comp. Physiol. A 178, 55-61.

Bandai, K., Arikawa, K. AND Eguchi, E. (1992). Localization of spectral receptors in the ommatidium of butterfly compound eye determined by polarization sensitivity. J. comp. Physiol. A 171, 289-297.

BRISCOE, A. D. (1998). Molecular diversity of visual pigments in the butterfly Papilio glaucus. Naturwissenschaften (in press).

Feiler, R., BJornson, R., Kirschfeld, K., Mismer, D., Rubin, G. M., Smith, D. P., Socolich, M. AND Zuker, C. S. (1992). Ectopic expression of ultraviolet-rhodopsins in the blue photoreceptor cells of Drosophila - visual physiology and photochemistry of transgenic animals. J. Neurosci. 12, 3862-3868.

Hariyama, T., Ozaki, K., Tokunaga, F. and Tsukahara, Y. (1993). Primary structure of crayfish visual pigment deduced from cDNA. FEBS Lett. 315, 287-292.

Mollon, J. D. ANd BowmaKer, J. K. (1992). The spatial arrangement of cones in the primate fovea. Nature 360, 677-679.

RöHLich, P., VANVEen, T. ANd SzÉL, A. (1994). Two different visual pigments in one retinal cone cell. Neuron 13, 1159-1166

SaKamoto, K., Hisatomi, O., ToKunaga, F. And Eguchi, E. (1996). Two opsins from the compound eye of the crab Hemigrapsus sanguineus. J. exp. Biol. 199, 441-450.

Satoh, T., Hisatomi, O. and Tokunaga, F. (1995). Anomalous expression of visual pigment genes in the killifish retina during development. Zool. Sci. 12 (Suppl.), 115.

Seki, T., Fujishita, S., Ito, M., Matsuoka, N. and Tsukida, K. (1987). Retinoid composition in the compound eyes of insects. Exp. Biol. 47, 95-103. 